Review

\title{
Development of Manganese-Based Nanoparticles as Contrast Probes for Magnetic Resonance Imaging
}

\author{
Zipeng Zhen and Jin Xie ${ }^{凶}$ \\ Department of Chemistry and Bio-Imaging Research Center (BIRC), University of Georgia, Athens, GA 30602
}

$\triangle$ Corresponding author: Dr. Jin Xie, Department of Chemistry, University of Georgia, 1001 Cedar Street, Athens, GA 30602, USA; Tel: 706-542-1933; Email: jinxie@uga.edu

(c) Ivyspring International Publisher. This is an open-access article distributed under the terms of the Creative Commons License (http://creativecommons.org/ licenses/by-nc-nd/3.0/). Reproduction is permitted for personal, noncommercial use, provided that the article is in whole, unmodified, and properly cited.

Received: 2011.08.26; Accepted: 2011.10.02; Published: 2012.01.01

\begin{abstract}
MRI is one of the most important imaging tools in clinics. It interrogates nuclei of atoms in a living subject, providing detailed delineation with high spatial and temporal resolutions. To compensate the innate low sensitivity, MRI contrast probes were developed and widely used. These are typically paramagnetic or superparamagnetic materials, functioning by reducing relaxation times of nearby protons. Previously, gadolinium(Gd)-based $T_{\text {, contrast probes }}$ were dominantly used. However, it was found recently that their uses are occasionally associated with nephrogenic system fibrosis (NSF), which suggests a need of finding alternatives. Among the efforts, manganese-containing nanoparticles have attracted much attention. By careful engineering, manganese nanoparticles with comparable $r$, relaxivities can be yielded. Moreover, other functionalities, be a targeting motif, a therapeutic agent or a second imaging component, can be loaded onto these nanoparticles, resulting in multifunctional nanoplatforms.
\end{abstract}

Key words: nanomedicine; MRI; manganese; contrast probes; nanoparticles; tumor imaging; theranostics.

\section{Introduction}

MRI is an imaging technique that interrogates nuclei of atoms inside a living body. It is one of the most frequently used imaging tools in daily clinics, allowing diagnosis to be performed in a noninvasive and real-time manner [1]. Its excellent soft tissue contrast makes it a complement to many other imaging modalities, such as computed tomography (CT), to afford both anatomical and functional information [2].

While blessed with an innate high resolution, the low sensitivity of MRI often times compromises diagnosis quality. In order to improve visibility, contrast agents of various forms have been developed and used [3-5]. These agents, functioning by shortening relaxation times of nearby protons, improve the contrast between lesions and normal tissues. This leads to better imaging quality and a reduced risk of misdiagnosis. In clinics, the most frequently used contrast agents are gadolinium (Gd)-based agents [6-8]. Due to an extremely high toxicity, Gd-based agents are always given in a chelated form. However, several cases of nephrogenic systemic fibrosis (NSF) have been reported recently on patients taking Gd-based contrast agents [9-11]. This once again raises concerns over the safety of this class of probes [12, 13] and therefore, spurring a new wave of exploit for non-Gd replacements [14]. As a metal ion with comparable $T_{1}$ contrast ability, $\mathrm{Mn}$ and its derivatives are regarded as promising alternatives. As a matter of fact, Mn-based agents have shown better performances than Gd-based ones in certain disease detec- 
tion, such as for pancreatic lesions [15].

The emergence of nanoparticle-based materials has added fuel to such an effort. With a size of several to hundreds of nanometers, nanomaterials are significantly larger than small molecules, allowing the loading of multiple copies of them. On the other hand, they are small enough to pass through many biological barriers and able to interact with a biological system at the molecular level. Through decades of research, it is believed that the technology has evolved to a level that allows the construction of nanoscale materials in a controlled manner. We can now synthesize nanoparticles, by chemical, physical or biological means, with accurate control over many parameters, including their size, shape, composition and surface nature. Tailoring these parameters, in turn, gives us the ability to tune particles' physical properties, such as their ability to induce contrast in MRI. Previously, iron oxide nanoparticles have been studied as $T_{2} / T_{2}{ }^{*}$ contrast agents [16-20], especially for imaging reticuloendothelial system (RES) organs like the liver, spleen and lymph nodes [4, 19]. However, iron oxide nanoparticles induce hypointensities. Such a negative contrast may be confused with areas of innate hypointensities, such as hemorrhage and blood clots, or interfered with magnetic susceptibility artifacts [21]. Both kinds of inferences can lead to inaccurate diagnosis. Mn nanoparticle-based contrast agents, on the other hand, induce more favorable hyperintensities on a $T_{1}$-weighted map [22-24]. The related exploits are more recent but have attracted much attention. In this review article, we will discuss the recent progress in this field, with an emphasis on how to engineer nanoparticles to achieve a higher $r_{1}$ relaxivity.

\section{Types of Mn-based nanoparticles}

\section{I Manganese oxide nanoparticles}

Many approaches have been developed to synthesize manganese oxide $\left(\mathrm{MnO}\right.$ or $\left.\mathrm{Mn}_{3} \mathrm{O}_{4}\right)$ nanoparticles. One common route is to heat up oleate-Mn in a high boiling point solvent (e.g. 1-octadecene) to induce nucleation and particle growth [25]. Such a method allows the preparation of nanoparticles with accurate size control. In one of these reports, it is shown that $\mathrm{MnO}$ nanoparticles from 7 to $25 \mathrm{~nm}$ can be prepared in large scale. One major disadvantage, however, is the water insolubility of as-synthesized products. Those nanoparticles are surface-passivated with a layer of oleic acid, and therefore, are highly hydrophobic. To address this issue, a surface modification step is needed, which transfers the as-synthesized nanoparticles to a water phase. In fact, this is a common problem shared with many other kinds of nanoparticles prepared through high temperature decomposition. Likewise, the phase transfer methods are in many cases interchangeable [19].

Unlike $T_{2} / T_{2}{ }^{*}$ contrast agents, the $T_{1}$ contrast agents need to have direct interaction with the surrounding water protons to affect their relaxation times. For nanospheres, it means that only Mn ions on the surface are effective. With the same amount of $\mathrm{Mn}$, smaller nanoparticles tend to have a larger surface percentage and therefore, higher $r_{1}$ relaxivities. Indeed, when using phospholipids as the coating materials to transfer Mn nanoparticles into an aqueous solution, it was found that the $7 \mathrm{~nm}$ formulation, the smallest among all tested, has the highest $r_{1}$. In a clinical 3.0 T magnet, $r_{1}$ relaxivities were evaluated to be $0.37,0.18,0.13$ and $0.12 \mathrm{mM}^{-1} \mathrm{~s}^{-1}$ for $\mathrm{Mn}$ nanoparticles with a diameter of 7, 15, 20 and $25 \mathrm{~nm}$, respectively. In a separate study, Huang et al. used manganese stearate as the precursor and prepared $\mathrm{MnO} / \mathrm{Mn}_{3} \mathrm{O}_{4}$ nanoparticles by hydrothermal decomposition. It was reported that nanoparticles down to $5 \mathrm{~nm}$ can be prepared by such a method, and the $r_{1}$ was found over $1 \mathrm{mM}^{-1} \mathrm{~s}^{-1}[26]$. Further down this path, Baek et al. prepared $\mathrm{MnO}$ nanoparticles of 2-3 nm using triethylene glycol as the solvent and D-glucuronic acid as the coating material. The resultant $\mathrm{MnO}$ nanoparticles can have an $r_{1}$ as high as 7.02 $\mathrm{mM}^{-1} \mathrm{~s}^{-1}[27]$.

Aside from the size effect, the coating material that stands between particle surface and the surroundings is considered another important factor. For instance, Huang et al. used a dopamine-plus-human serum albumin (HSA) bilayer coating to make $\mathrm{MnO}$ nanoparticles water soluble [23]. In the first step, dopamine, with its adjacent hydroxyl groups, tightly bound with surface Mn to replace the original oleate coating. Subsequently, a layer of HSA was adsorbed onto the nanoparticle surface to bring in sufficient water dispensability (Figure 1). From the same batch of as-synthesized $\mathrm{MnO}$ nanoparticles, they then used both dopamine-plus-HSA and phospholipids to make $\mathrm{MnO}$ nanoparticles water soluble. It was found that particles yielded from the former strategy induced an $r_{1}$ that is over five times higher than from the latter, phospholipid-coated one (1.97 vs. $0.37 \mathrm{mM}^{-1} \mathrm{~s}^{-1}$ for 20 $\mathrm{nm} \mathrm{MnO}$ nanoparticles). Although the detailed mechanism is unknown, it is believed that the more hydrophilic bilayer is essential. Unlike in the case of phospholipids, where a hydrophobic zone exists between particle surface and the aqueous surroundings, both dopamine and HSA are hydrophilic materials and allows efficient water penetration. 

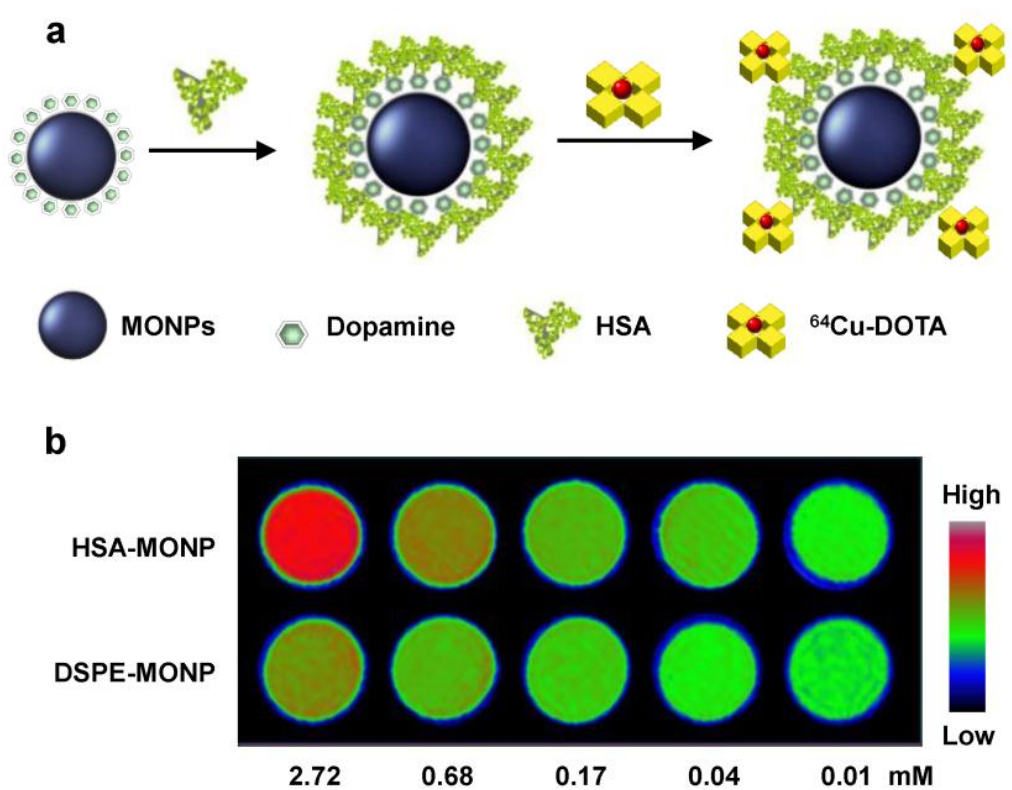

Figure I. a) Schematic illustration of the dopamine-plus-HSA coating strategy. b) Phantom studies with nanoparticles coated with HSA (the first row) and phospholipids (the second row). At the same Mn concentration, HSA coated MnO nanoparticles showed much higher hyperintensities. Reproduced with permission from Ref. [23].

The advanced synthetic technology also allows the preparation of composite nanoparticles. For instance, the synthesis of $\mathrm{Au}-\mathrm{MnO}$ nanoparticles with either a flower-like shape or a dumbbell-like shape was reported $[28,29]$. It typically starts with $\mathrm{Au}$ nanoparticles, and subsequently grows $\mathrm{MnO}$ petals on top of these seeds. Schladt et al. used a multidentate copolymer carrying catechol anchors and PEG linkers to replace the original hydrophobic coating on $\mathrm{Au}-\mathrm{MnO}$ nanoflowers. The resulting nanoparticles are water soluble and showed an $r_{1}$ of $0.224 \mathrm{mM}^{-1} \mathrm{~s}^{-1}$. Such a relatively low $r_{1}$ can be explained by the fact that a large part of $\mathrm{MnO}$ is hinged with $\mathrm{Au}$ nanoparticles and is not accessible to water. An advantage of using such a composite nanostructure is that it has two surface types and can be functionalized dually. While the $\mathrm{MnO}$ have high affinity with catechol and its derivatives, the $\mathrm{Au}$ particles can be easily modified with thiolated species. In theory, two types of functionalities can be loaded without causing cross-species interference. As a proof-of-concept study, 4-chloro-7nitrobenzofurazan (NBD) and a Texas red tagged 24-mer oligonucleotide were loaded separately onto the $\mathrm{MnO}$ and $\mathrm{Au}$ surface of $\mathrm{Au}-\mathrm{MnO}$ nanoflowers [29].

\subsection{Hollow manganese oxide nanoparticles}

As mentioned above, an increased nanoparticle surface percentage can lead to a higher $r_{1}$. Instead of reducing the nanoparticle size, it is also possible to achieve this goal by converting particles into a hollowed structure. It was reported that treating $\mathrm{MnO}$ nanoparticles at high temperature $\left(300^{\circ} \mathrm{C}\right)$ in technical grade trioctylphosphine oxide can lead to the formation of $\mathrm{MnO}$ nanohollows. It was later revealed that the impurities, mostly alkylphosphonic acid, were playing a role as an etching agent [30]. Further characterizations found that an inward diffusion of phosphorus and an outward diffusion of manganese took place in the intermediate stages during the etching, a so-called Kirkendall effect. This generated a void within the original nanoparticle (Figure 2). The resultant nanostructures retained the size and shape uniformity of the original nanocrystals but have significantly increased water accessibility. For $20 \mathrm{~nm}$ $\mathrm{MnO}$ nanoparticles, this treatment can increase $r_{1}$ from 0.353 to $1.15 \mathrm{mM}^{-1} \mathrm{~s}^{-1}$.

An alternative way of forming $\mathrm{MnO}$ hollowed nanostructures is to incubate water soluble $\mathrm{MnO}$ nanoparticles in a phthalate buffer ( $\mathrm{pH} 4.6$, r.t.) for $12 \mathrm{~h}$. This will not affect the coating but will etch away part of the $\mathrm{MnO}$ interior to induce a void. For $20 \mathrm{~nm} \mathrm{MnO}$ $\mathrm{NPs}$, it was reported that an $r_{1}$ as high as $1.42 \mathrm{mM}^{-1} \mathrm{~s}^{-1}$ can be achieved [31].

\subsection{Silica-coated manganese oxide nanoparticles}

Instead of adopting an organic coating, $\mathrm{MnO}$ nanoparticles can also be coated with a layer of silica. 
There are at least two folds of merits with silica coatings. First, silica is a biocompatible material. Second, silica coating allows easy functionality loading, either onto the particle surface or into the silica framework. Dyes, photosensitizers and many other kinds of functionalities can be pre-conjugated with silane derivatives, such as 3-aminopropyltrimethoxysilane (APTMS), and loaded at high rate into silica matrices. However, intact silica coating is too dense to allow efficient water infiltration. To overcome this issue, Peng et al. developed an etching process to induce a mesoporous structure and to improve water permeability. It was achieved by incubating silica-coated $\mathrm{MnO}$ nanoparticles with ethylacetate and sodium hydroxide at $60^{\circ} \mathrm{C}$. Such a mesoporous structure allows water molecules to move in through the pores and interact with the surface $\mathrm{Mn}\left(\mathrm{MnO} @ \mathrm{mSiO}_{2}\right)$. More excitingly, further treatment under this condition can lead to corrosion of the imbedded $\mathrm{MnO}$ cores, generating hollowed cores $\left(\mathrm{H}-\mathrm{MnO} @ \mathrm{mSiO}_{2}\right)$. As mentioned above, this hollowed structure can further improve $r_{1}$ relaxivites. Indeed, $\mathrm{MnO}$ nanoparticles with intact silica coatings only have an $r_{1}$ of $0.07 \mathrm{mM}^{-1} \mathrm{~s}^{-1}$. Such a figure was found increased to $0.16 \mathrm{mM}^{-1} \mathrm{~s}^{-1}$ for $\mathrm{MnO} @ \mathrm{mSiO}_{2}$ and $0.2 \mathrm{mM}^{-1} \mathrm{~s}^{-1}$ for $\mathrm{H}-\mathrm{MnO} @ \mathrm{mSiO}_{2}$ [32].

In a separate study, Kim et al. used a different approach to induce porous structure. They introduced cetyl trimethylammonium bromide (CTAB) along with tetraethyl orthosilicate (TEOS) during a silica sol-gel reaction process. CTAB served as the organic structure-directing template [21]. Later on, when incubated in refluxing ethanol solutions $(\mathrm{pH}=2.4)$, such a CTAB template was removed, leaving pores in the silica matrices (Figure 3). Like in the above case, such a treatment in acid environment also led to the formation of a hollowed nanostructure. For $15 \mathrm{~nm} \mathrm{MnO}$ nanoparticles, it was reported that an $r_{1}$ of 1.72 $\mathrm{mM}^{-1} \mathrm{~s}^{-1}$ can be achieved. The resulting mesoporous silica-coated hollow manganese oxide (HMnO@mSiO2) nanoparticles are able to efficiently label adipose-derived mesenchymal stem cells (MSCs) and to facilitate the tracking of MSCs in vivo by MRI (Figure 3).

Compared with $\mathrm{MnO}$ nanohollows with an organic coating, it is obvious that the silica coated ones have slightly lower $r_{1}$ relaxivities. This is not surprising considering a higher surface coverage rate of the latter. On the other hand, silica coating affords a scaffold effect which stabilizes the nanohollow structure. This brings a better morphology control and less worry on the nanostructure collapse. Last but not least, the well-established silica engineering techniques allow the easy loading of various types of functionalities. Organic dyes, inorganic fluorophores, small molecule drugs, peptides, antibodies and siRNA have all been loaded onto silica coated nanoparticles, independent of the nature of cores $[19,33]$.
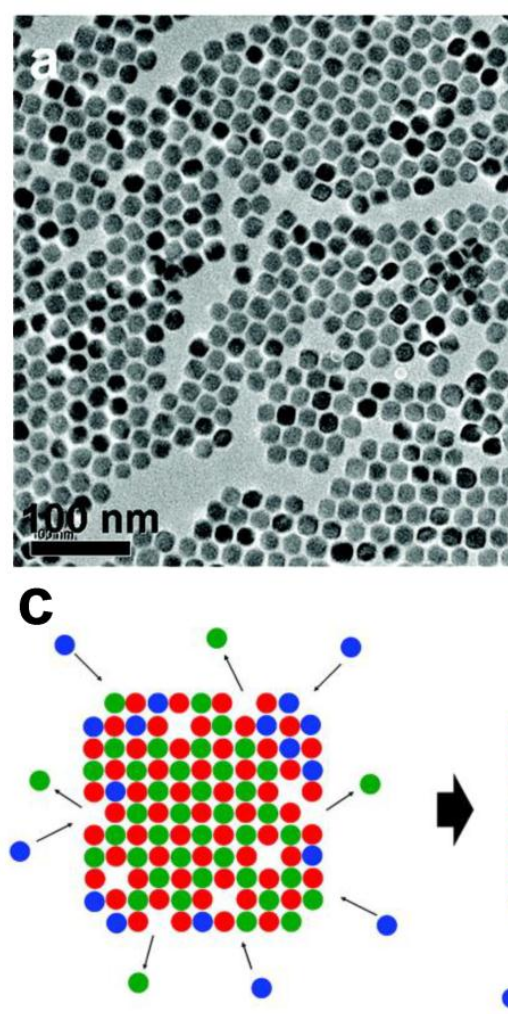
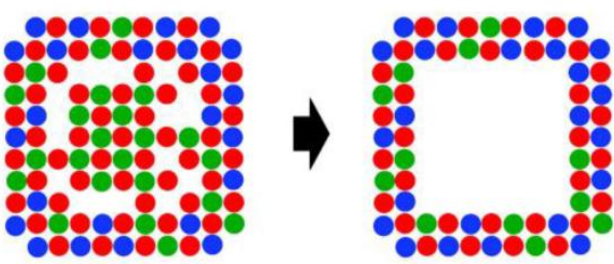

Figure 2. TEM images taken a) before and b) after the etching process. Voids were generated inside the nanostructures. c) Suggested mechanism for the generation of voids. An inward diffusion of phosphorus and an outward diffusion of manganese took place at the same time, leading to the formation of a void in the nanostructure. Reproduced with permission from Ref. [30]. 

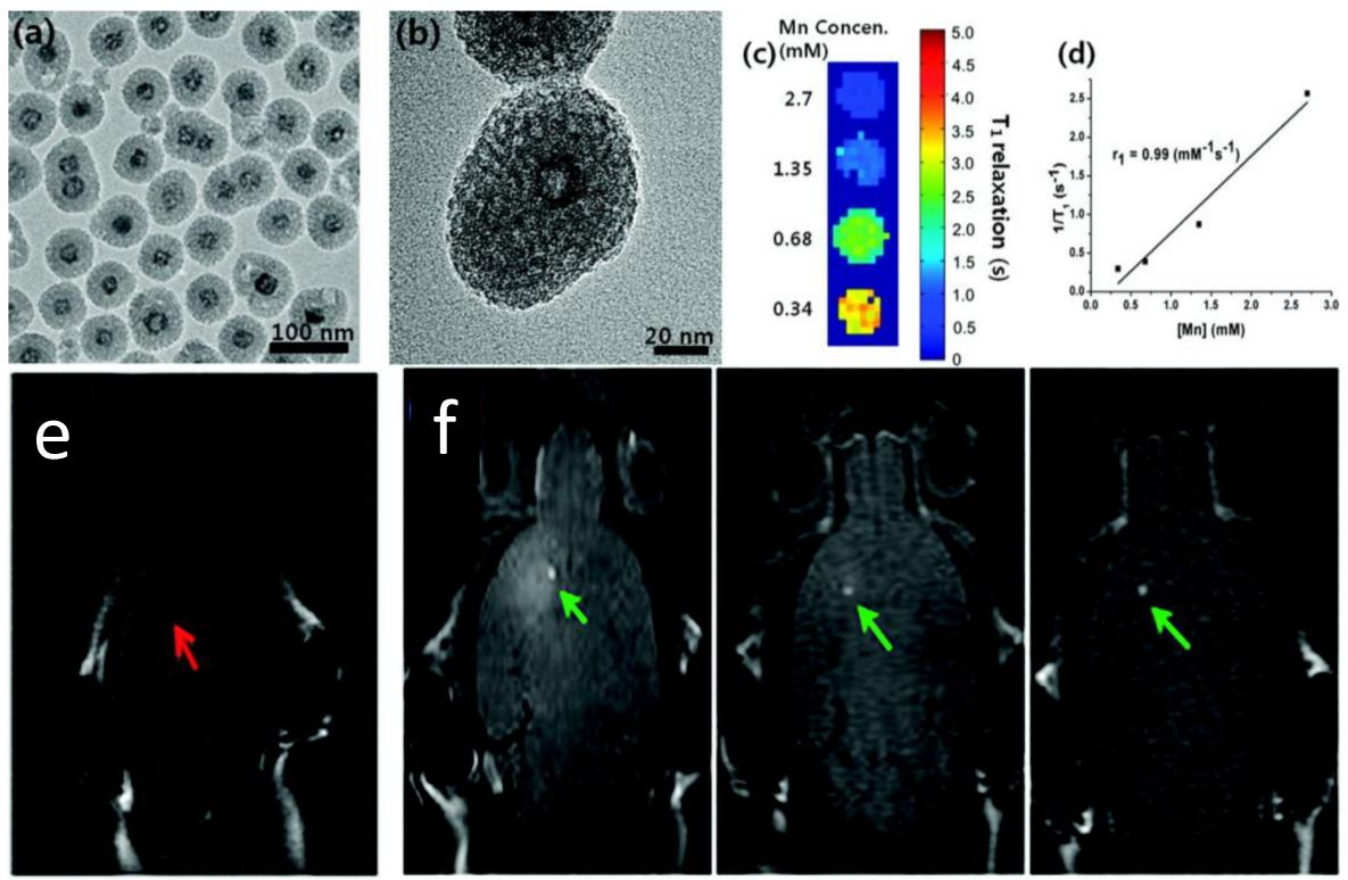

Day 1

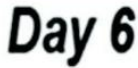

Day 14

Figure 3. Characterizations of $\mathrm{HMnO} @ m S i O_{2}$ nanoparticles. a) TEM image. b) HRTEM image of a single nanoparticle. c) $T_{1}$ map of $\mathrm{HMnO} @ \mathrm{mSiO}_{2}$ nanoparticles suspended in water at II.7 T. d) Plot of I/T, versus Mn concentration. The slope indicates the specific relaxivity $\left(r_{1}\right)$. e-f) In vivo MRI of transplanted MSCs. e) No hyperintense signal (red arrow) was detected in mouse transplanted with unlabeled MSCs. f) Hyperintense signals (green arrows) were detected in mouse transplanted with $\mathrm{HMnO} @ \mathrm{mSiO}_{2}$-labeled $\mathrm{MSCs}$ and were still detectable 14 days after the injection. Reproduced with permission from Ref. [2I].

\subsection{Mn-doped nanoparticles and nanoscale met- al-organic frameworks}

Instead of engineering manganese oxide nanoparticles, it is also possible to arrive at Mn-based probes by doping free Mn cations or incorporating Mn complexes into other nanoscaffolds. Previously, Niesman et al. has loaded $\mathrm{MnCl}_{2}$ into liposomes and used them as liver specific contrast agents [34]. It was shown from toxicity studies that the effective dose of such a complex was 7 to 11 times lower than the LD50 of free $\mathrm{MnCl}_{2}$. In rats with implanted liver tumors, those particles induced a two- to three-fold increase in the relaxation rate of liver while having little effect on the tumor relaxation rate, making this formulation a potential contrast agent for liver metastasis imaging [34]. In a separate study, Pan et al. loaded Mn(III), in the form of a porphyrin complex, onto a nanobialy constructed by amphiphilic branched polyethylenimine (PEI) [35]. Such an amphiphilic PEI was achieved from alkylating PEI10k with linoleic acid. The resultant PEI can self-assemble into a toroidal shape with low polydisperisity. Mn(III)protoporphyrin chloride (Mn-PPC) can be easily in- corporated into such an inverted micellar structure (Figure 4). Most Mn-PPC was found at the surface of the nanostructure, a feature that is essential for water accessibility. Under a $1.5 \mathrm{~T}$ magnet, an $r_{1}$ of $3.7 \mathrm{mM}^{-1} \mathrm{~s}^{-1}$ was recorded. Such a nanobialy structure can be further loaded with small drug molecules, such as camptothecin or doxorubicin, with high efficiency (98 $\pm 0.1 \%$ and $99 \pm 0.1 \%$ for doxorubicin and camptothecin, respectively). It is therefore regarded as a potential theranostic nanoplatform, having both diagnostic and therapeutic values. Another example is Mn-oleate based nanocolloids reported by Pan et al. In brief, Mn-oleate was suspended with polysorbates (e.g. sorbitan sesquioleate) and homogenized with a mixture of surfactants, mainly comprised of phosphatidylcholine (PC) ( $\sim 90 \mathrm{~mol} \%)$ and biotin-caproyl-PE ( 1\%). This leads to the formation of a nanocolloid with a hydrodynamic size of $134 \pm 2 \mathrm{~nm}$. Under a $3.0 \mathrm{~T}$ magnet, the $r_{1}$ was evaluated to be $14.6 \pm 1.1$ $\mathrm{mM}^{-1} \mathrm{~s}^{-1}[36]$.

Recently, silicon-based quantum dots (QDs) have attracted much attention. Compared to the previously used QDs, Si QDs are more favorable for their 
better biocompatibility and biodegradability [37]. It was reported recently that Mn can be doped into such a system to achieve probes with MRI/optical dual functionalities. For preparation, a high-energy mill was used to ball-mill mixtures of $\mathrm{NaH}, \mathrm{Si}$ and $\mathrm{Mn}$ powders with an appropriate molar ratio. Such a mixture was placed into an alumina crucible with a lid which was placed into a quartz tube. It was then heated at $420^{\circ} \mathrm{C}$ for 2 days followed by heating at $500^{\circ} \mathrm{C}$ for 1 additional day under flowing nitrogen. The yielded sodium silicide (Mn-doped) was used as a precursor and heated to reflux for $2 \mathrm{~h}$ in DMF with the presence of $\mathrm{NH}_{4} \mathrm{Br}$ and allylamine. The resultant nanoparticles can be dispersed in water and can be further stabilized by adding a dextran sulfate or dextran coating. Two-photon excitation found a peak at $790 \mathrm{~nm}$, which is in the near-infrared region and therefore, has optimum tissue penetration. These nanoparticles demonstrated a very high $r_{1}$ relaxivity at $25.50 \pm 1.44 \mathrm{mM}^{-1} \mathrm{~s}^{-1}[38]$.

Recently, Mn-containing metal-organic framework was constructed by Taylor et al. and was studied as an MRI contrast probe [39]. Unlike the above-mentioned nanocrystals, such a nanostructure is not metal-oxide-based. It was synthesized by using either terephthalic acid (BDC) or trimesic acid (BTC) as a bridging ligand. Then through a reverse-phase microemulsion process, $\mathrm{Mn}$ (II) complexed with the bridging ligands and grew into a nanorod structure (Figure 5). These nanoparticles, too, can be easily coated with silica and further loaded with other functionalities. The resultant nanoparticles demonstrated good $r_{1}$ relaxivities $\left(5.5 \mathrm{mM}^{-1} \mathrm{~s}^{-1}\right.$ under $3.0 \mathrm{~T}$ and 4.6 $\mathrm{mM}^{-1} \mathrm{~s}^{-1}$ under $\left.9.4 \mathrm{~T}\right)$.

\section{Multifunctional manganese oxide nano- particles}

Like many other nanoplatforms, Mn particles can be coupled with different kinds of functionalities and upgraded to multifunctional nanogadgets. For instance, it is possible to conjugate a targeting motif, such as a peptide [40,41] or an antibody [4], onto nanoparticles to gift them targeting specificity. In one of these studies, MnO nanoparticles were coupled with Herceptin, an antibody that targets human epidermal growth factor receptor 2 (HER2) [42, 43]. The conjugates, after i.v. injected, were able to home to breast cancer metastasis in the brain. It is believed that the blood brain barrier (BBB), which blocks the infiltration of particles into the brain, was destructed at the tumor site. While both non-functionalized and functionalized nanoparticles can penetrate BBB, only the Herceptin conjugated ones were able to home to the tumor sites.
It is also possible to put other imaging components onto nanoparticles to achieve multimodality imaging probes. It was mentioned above that Mn can be doped into a silicon QD matrix to achieve dually functional imaging probes with both MRI and optical imaging capabilities. From an in vitro analysis, they found that these nanoparticles can be efficiently taken up by macrophages without dramatically affecting the cells' viability. The particle-labeled cells can be easily recognized by both MRI and two-photon optical imaging, suggesting their potential as cell labeling reagents [38].

Therapeutics of various forms can also be loaded onto Mn-based nanoplatforms. Choi et al. loaded doxorubicin, via electrostatic interaction, onto silica coated $\mathrm{MnO}$ nanoparticles. These nanoparticles were further loaded into a 3-D poly(propylene fumarate) (PPF) scaffold. It was found that the release of doxorubicin into the surroundings can be tracked by both MR and optical imaging [44]. Shin et al. demonstrated that doxorubicin can be loaded into hollow $\mathrm{MnO}$ nanoparticles at $202 \mu \mathrm{g} /$ mole particle, a rate which is much higher than into intact $\mathrm{MnO}$ nanoparticles (58 $\mu \mathrm{g} /$ mole particle). Doxorubicin is amphiphatic, and in principle a hydrophobic molecule. It is believed that the hydrophobic-hydrophobic interaction between doxorubicin and the oleic acid coating is the main cause of the loading. With higher oleic acid coverage, the hollowed nanostructure affords a higher coating capacity [31].

In another important study, Viglianti et al. loaded both $\mathrm{MnSO}_{4}$ and doxorubicin into a liposome formulation (Figure 6). It is believed that two doxorubicin molecules are coordinated to each $\mathrm{Mn}^{2+}$ cation within the liposome, where water molecules have limited accessibility. The release of Mn into the surroundings is found associated with significant $T_{1}$ shortening and this feature was studied as a means to monitor the in vivo drug release. The drug release was triggered by inducing heat to tumors. When using a thermal sensitive liposome formulation, it was found clearly that the drug release occurred preferentially at the periphery of tumors (Figure 6). This pattern was concordant with the release temperature of the formulation $\left(39-40^{\circ} \mathrm{C}\right)$. As a control, a nonthermally sensitive formulation was also tested. While selective tumor accumulation was also observed, a heterogeneous drug distribution pattern was found in such a case [45]. Later on, the same group studied whether the timing of liposome administration is critical to the drug release. It was found that doxorubicin was accumulated more rapidly and efficiently if the formulation was injected during rather than before the hyperthermia [46]. 


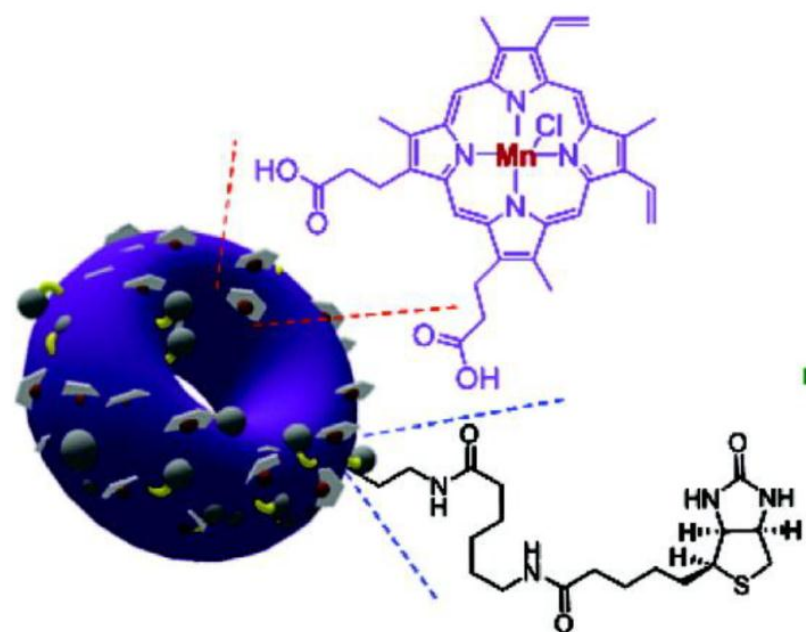

Mn(III)-labeled nanoparticles targeted to human plasma clot

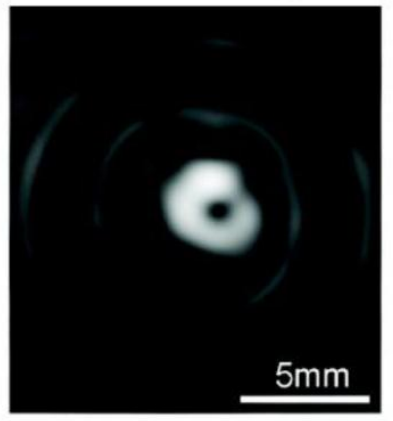

Manganese based MR imaging

Figure 4. Linoleic acid modified PEl can self-assemble into a toroidal shape nanostructure. Mn(III)-protoporphyrin chloride (Mn-PPC) was able to be incorporated into such an inverted micellar structure. Under a $1.5 \mathrm{~T}$ magnet, an $r_{1}$ of $3.7 \mathrm{mM}^{-1} \mathrm{~s}^{-1}$ was recorded. Reproduced with permission from Ref. [35].
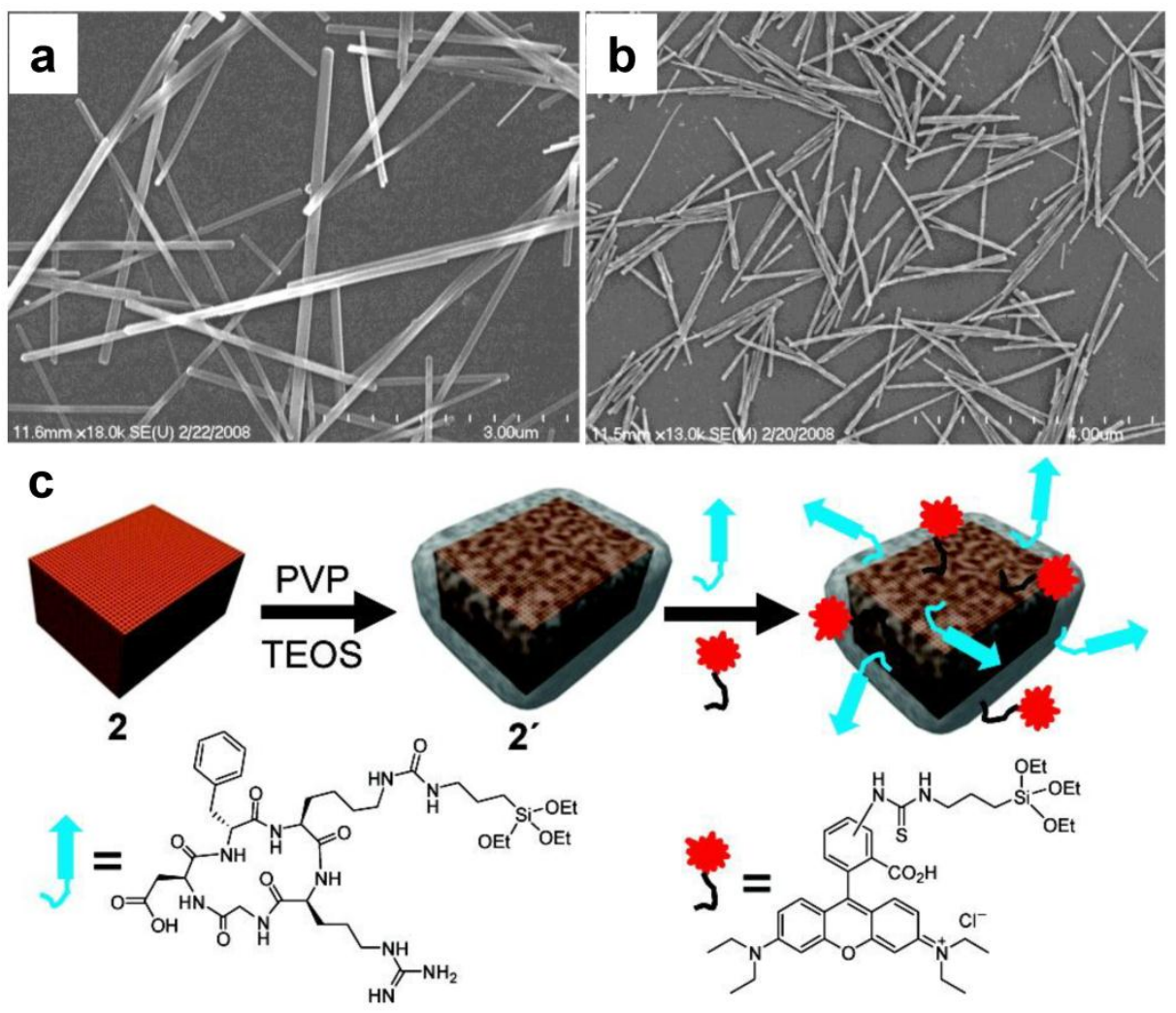

Figure 5. SEM images of a) $\mathrm{Mn}(\mathrm{BDC})\left(\mathrm{H}_{2} \mathrm{O}\right)_{2}$ nanorods and b) $\mathrm{Mn}_{3}(\mathrm{BTC})_{2}\left(\mathrm{H}_{2} \mathrm{O}\right)_{6}$ spiral nanorods. c) Nanorods can be surface-functionalized with PVP and then coated with a thin layer of silica. Other functionalities, such as peptides and dye molecules, can be as well loaded onto the silica coating. Reproduced with permission from Ref. [39]. 

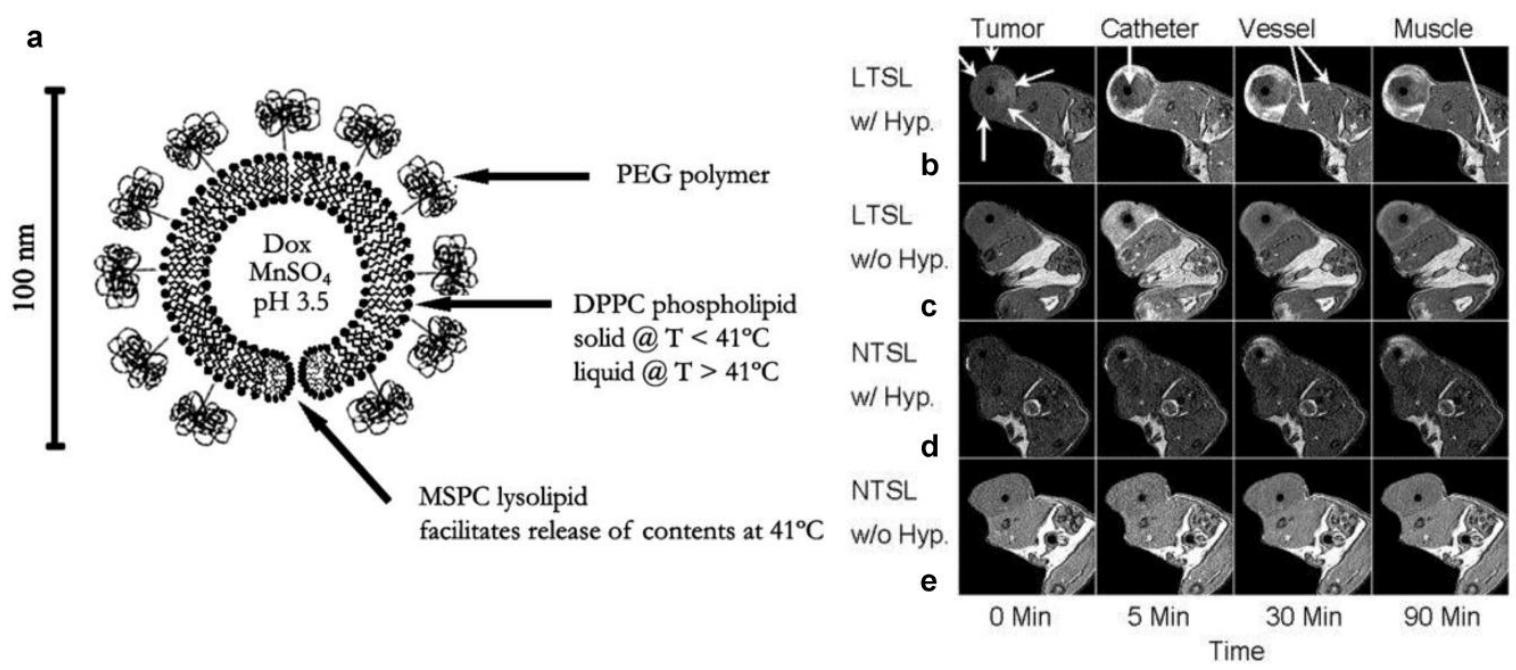

Figure 6. a) Schematic depiction of a temperature-sensitive liposome containing doxorubicin (Dox) and contrast agent (manganese sulfate, $\mathrm{MnSO}_{4}$ ). The phospholipid bilayer undergoes a main melting phase transition at $4 \mathrm{I}^{\circ} \mathrm{C}$, which leads to the release of contents. DPPC = I,2 dipalmitoyl-sn-glycerol-3-phosphocholine; MSPC = I-stearoyl-2-hydroxy-sn-glycero-3phosphocholine. b-e) MRI axial images of rats with transplanted flank fibrosarcomas treated with doxorubicin/Mn containing liposomes. b) LTSL with HT, c) LTSL without HT, d) NTSL with HT, and e) NTSL without HT from 0-90 min. LTSL = lysolipid-based temperature-sensitive liposomes; HT = hyperthermia; NTSL = non-temperature-sensitive liposomes. Reproduced with permission from Ref. [45] and [46].

Very recently, Bae et al. demonstrated the possibility of loading siRNA onto hollow $\mathrm{MnO}$ nanoparticles. They coupled 3,4-dihydroxy-Lphenylalanine with polyethylenimine(PEI) and used the resultant conjugate as an adhesive moiety to modify the hollow MnO particle surface. Herceptin was loaded via chemical conjugation. On the other hand, siRNA was loaded by electrostatically interacting with the polycation coating. They demonstrated at the in vitro level that the resultant conjugates can selectively target HER2 overexpressed cells and induce silencing of the target gene [47].

\section{Toxicity}

One major concern of using Mn-based nanoparticles is their cellular toxicity. Manganese is considered as an essential metal for biological systems, working as a cofactor for a number of key enzymes $[48,49]$. However, high concentrations of Mn is associated with neurotoxicity. This includes progressive and permanent neurodegenerative disorders, altogether termed as manganism, which resembles Parkinsonism in many ways $[50,51]$. Nonetheless, Mn formulations have been used frequently in clinics to help improve the quality of MR imaging. $\mathrm{MnCl}_{2}$, for instance, is an FDA approved contrast agent. Due to its ability to enter excitable cells through voltage-gated calcium channels, it is widely used in MRI to demarcate active regions of the brain $[52,53]$. In small animal studies, it was recommended that doses higher than $93 \mathrm{mg} / \mathrm{kg}$ and $38 \mathrm{mg} / \mathrm{kg}$ should not be used for rats and mice, respectively [54]. However, a much higher dose was used with manageable adverse effects $[55,56]$. Chelated $\mathrm{Mn}$ is considered much safer than free $\mathrm{Mn}(\mathrm{II})$. Mangafodipir trisodium (MnDnnP), for instance, is another FDA approved formulation, used extensively in hepatobiliary MRI $[57,58]$. It is believed that its structural similarity to vitamin B6 is one of the main factors for its high hepatocyte uptake [59]. It is noted, however, that $\mathrm{Mn}$ (II) can be released from the complex through metabolism. Those released cations accumulate in the liver, pancreas and cardiac muscle and cause hyperintensities. At suggested does, however, those released Mn will not cause dramatic side effects.

On the other hand, relatively little attention has been paid to the toxicity of Mn-doped nanoparticles. So far, most research in this area has been satisfied with in vitro viability assays. It was claimed by many that Mn-doped nanoparticles, be a silica matrix or a metal-organic framework, are safer than Mn chelators, since the Mn are less likely released during circulation. However, these nanoparticles, with a larger size, cannot be secreted through renal clearance and may stay in a living subject for a long period of time. What is the metabolism route and how the daughter compounds interact with a biological system is largely unknown at this point. A similar issue was found in 
the research on manganese oxide-based formulations. One common argument is that $\mathrm{Mn}$ is in a crystallized form in $\mathrm{MnO}$ or $\mathrm{Mn}_{3} \mathrm{O}_{4}$ nanoparticles and does not easily fall off. Indeed, viability studies have shown that cells can tolerate these nanoparticles to a certain degree. $\mathrm{MnO}$ nanoparticles have even been used for labeling and tracking transplanted cells [60]. However, cytotoxicity studies provide limited information on the long-term impact. At the end of the analysis (typically within $72 \mathrm{~h}$ ), many nanoparticles are still in the process of decomposing in an acidic endosome/lysosome environment. Systematic studies are needed to better understand the detailed particle metabolism and their long-term effects to a living subject, especially from a neurotoxicity perspective.

\section{Conclusions and perspective}

Mn-based nanoparticles are a relative new class of materials. Unlike the other well-studied nanoparticles, such as iron oxides [61], research on Mn-based nanoparticles is at a relatively early stage. Much more work has to be done to promote this new class of materials in a nanomedicine context. Several key parameters include their $T_{1}$ contrast ability and their distribution in a living subject, either with or without a targeting motif.

To estimate their translation potential, more efforts should be put on accessing the biosafety of Mn-based nanoparticles. The current analysis-of-standard is in vitro viability assay [62]. At this stage, little is known about the nanoparticles' metabolism and the long-term side effects to a living subject. Also, it is worthwhile to perform a side-by-side comparison with both Gd- and iron oxide-based formulations to assess the advantages of Mn-based nanoparticles, if there is any.

\section{Acknowledgements}

Supported by NIH grant R00 4R00CA153772.

\section{Conflict of Interest}

The authors have declared that no conflict of interest exists.

\section{References}

1. Cleary K, Peters TM. Image-guided interventions: technology review and clinical applications. Annu Rev Biomed Eng. 2010; 12: $119-42$.

2. Alger JR, Frank JA. The utilization of magnetic resonance imaging in physiology. Annu Rev Physiol. 1992; 54: 827-46.

3. Xie J, Liu G, Eden HS, Ai H, Chen X. Surface-Engineered Magnetic Nanoparticle Platforms for Cancer Imaging and Therapy. Acc Chem Res. 2011;44(10):883-92.

4. Xie J, Huang J, Li X, Sun S, Chen X. Iron oxide nanoparticle platform for biomedical applications. Curr Med Chem. 2009; 16: 1278-94.
5. Bogdanov A, Jr., Mazzanti ML. Molecular magnetic resonance contrast agents for the detection of cancer: past and present. Semin Oncol. 2011; 38: 42-54.

6. Niendorf HP, Felix R, Laniado M, Schorner W, Kornmesser W, Claussen C. Magnetic resonance imaging of intracranial tumors using gadolinium-DTPA. Initial experience with fast imaging. Acta Radiol Suppl. 1986; 369: 561-3.

7. Girardot C, Boukobza M, Lamoureux JP, Sichez JP, Capelle L, Zouaoui A, et al. Choroid plexus papillomas of the posterior fossa in adults: MR imaging and gadolinium enhancement. Report of four cases and review of the literature. J Neuroradiol. 1990; 17: 303-18.

8. Pishko GL, Astary GW, Mareci TH, Sarntinoranont M. Sensitivity analysis of an image-based solid tumor computational model with heterogeneous vasculature and porosity. Ann Biomed Eng. 2011;39: 2360-73.

9. Sieber MA, Steger-Hartmann $T$, Lengsfeld $P$, Pietsch $H$. Gadolinium-based contrast agents and NSF: evidence from animal experience. J Magn Reson Imaging. 2009; 30: 1268-76.

10. Prince MR, Zhang HL, Prowda JC, Grossman ME, Silvers DN. Nephrogenic systemic fibrosis and its impact on abdominal imaging. Radiographics. 2009; 29: 1565-74.

11. Idee JM, Port M, Dencausse A, Lancelot E, Corot C. Involvement of gadolinium chelates in the mechanism of nephrogenic systemic fibrosis: an update. Radiol Clin North Am. 2009; 47: 855-69.

12. Grobner T. Gadolinium--a specific trigger for the development of nephrogenic fibrosing dermopathy and nephrogenic systemic fibrosis? Nephrol Dial Transplant. 2006; 21: 1104-8.

13. Marckmann P, Skov L, Rossen K, Dupont A, Damholt MB, Heaf JG, et al. Nephrogenic systemic fibrosis: suspected causative role of gadodiamide used for contrast-enhanced magnetic resonance imaging. J Am Soc Nephrol. 2006; 17: 2359-62.

14. Pan C, Guan J, Lin M. A multidisciplinary approach to identify a degradation product in a pharmaceutical dosage form. J Pharm Biomed Anal. 2011; 54: 855-9.

15. Diehl SJ, Lehmann KJ, Gaa J, McGill S, Hoffmann V, Georgi M. MR imaging of pancreatic lesions - Comparison of manganese-DPDP and gadolinium chelate. Invest Radiol. 1999; 34: 589-95.

16. Kessinger CW, Togao $\mathrm{O}$, Khemtong $\mathrm{C}$, Huang G, Takahashi M, Gao J. Investigation of In Vivo Targeting Kinetics of alpha(v)beta(3)-Specific Superparamagnetic Nanoprobes by Time-Resolved MRI. Theranostics. 2011; 1: 263-73.

17. Quan Q, Xie J, Gao H, Yang M, Zhang F, Liu G, et al. HSA Coated Iron Oxide Nanoparticles as Drug Delivery Vehicles for Cancer Therapy. Mol Pharm. 2011; 8:1669-76.

18. Huang J, Bu L, Xie J, Chen $\mathrm{K}$, Cheng $\mathrm{Z}, \mathrm{Li} \mathrm{X}$, et al. Effects of nanoparticle size on cellular uptake and liver MRI with polyvinylpyrrolidone-coated iron oxide nanoparticles. ACS Nano. 2010; 4: 7151-60.

19. Xie J, Lee S, Chen X. Nanoparticle-based theranostic agents. Adv Drug Deliv Rev. 2010; 62: 1064-79.

20. Xie J, Chen K, Huang J, Lee S, Wang J, Gao J, et al. PET/NIRF/MRI triple functional iron oxide nanoparticles. Biomaterials. 2010; 31: 3016-22.

21. Kim T, Momin E, Choi J, Yuan K, Zaidi H, Kim J, et al. Mesoporous silica-coated hollow manganese oxide nanoparticles as positive T1 contrast agents for labeling and MRI tracking of adipose-derived mesenchymal stem cells. J Am Chem Soc. 2011; 133: 2955-61.

22. Xing R, Liu G, Quan Q, Bhirde A, Zhang G, Jin A, et al. Functional $\mathrm{MnO}$ nanoclusters for efficient siRNA delivery. Chem Commun (Camb). 2011; 47:12152-4.

23. Huang J, Xie J, Chen $\mathrm{K}, \mathrm{Bu} \mathrm{L}$, Lee S, Cheng $\mathrm{Z}$, et al. HSA coated $\mathrm{MnO}$ nanoparticles with prominent MRI contrast for tumor imaging. Chem Commun (Camb). 2010; 46: 6684-6.

24. Xing R, Zhang F, Xie J, Aronova M, Zhang G, Guo N, et al. Polyaspartic Acid Coated Manganese Oxide Nanoparticles for Efficent Liver MRI. Nanoscale. 2011; epub. 
25. Park J, An K, Hwang Y, Park JG, Noh HJ, Kim JY, et al. Ultra-large-scale syntheses of monodisperse nanocrystals. Nat Mater. 2004; 3: 891-5.

26. Huang CC, Khu NH, Yeh CS. The characteristics of sub $10 \mathrm{~nm}$ manganese oxide $\mathrm{T} 1$ contrast agents of different nanostructured morphologies. Biomaterials. 2010; 31: 4073-8.

27. Baek MJ, Park JY, Xu W, Kattel K, Kim HG, Lee EJ, et al. Water-soluble $\mathrm{MnO}$ nanocolloid for a molecular T1 MR imaging: a facile one-pot synthesis, in vivo T1 MR images, and account for relaxivities. ACS Appl Mater Interfaces. 2010; 2: 2949-55.

28. Choi SH, Na HB, Park YI, An K, Kwon SG, Jang Y, et al. Simple and generalized synthesis of oxide-metal heterostructured nanoparticles and their applications in multimodal biomedical probes. J Am Chem Soc. 2008; 130: 15573-80.

29. Schladt TD, Shukoor MI, Schneider K, Tahir MN, Natalio F, Ament I, et al. Au@MnO nanoflowers: hybrid nanocomposites for selective dual functionalization and imaging. Angew Chem Int Ed Engl. 2010; 49: 3976-80.

30. An K, Kwon SG, Park M, Na HB, Baik SI, Yu JH, et al. Synthesis of uniform hollow oxide nanoparticles through nanoscale acid etching. Nano Lett. 2008; 8: 4252-8.

31. Shin J, Anisur RM, Ko MK, Im GH, Lee JH, Lee IS. Hollow manganese oxide nanoparticles as multifunctional agents for magnetic resonance imaging and drug delivery. Angew Chem Int Ed Engl. 2009; 48: 321-4.

32. Peng YK, Lai CW, Liu CL, Chen HC, Hsiao YH, Liu WL, et al. A new and facile method to prepare uniform hollow $\mathrm{MnO} /$ functionalized $\mathrm{mSiO}$ core/shell nanocomposites. ACS Nano. 2011; 5: 4177-87.

33. Liu Y, Lou C, Yang H, Shi M, Miyoshi H. Silica nanoparticles as promising drug/gene delivery carriers and fluorescent nano-probes: recent advances. Curr Cancer Drug Targets. 2011; 11: 156-63.

34. Niesman MR, Bacic GG, Wright SM, Swartz HJ, Magin RL. Liposome encapsulated $\mathrm{MnCl} 2$ as a liver specific contrast agent for magnetic resonance imaging. Invest Radiol. 1990; 25: 545-51.

35. Pan D, Caruthers SD, Hu G, Senpan A, Scott MJ, Gaffney PJ, et al. Ligand-directed nanobialys as theranostic agent for drug delivery and manganese-based magnetic resonance imaging of vascular targets. J Am Chem Soc. 2008; 130: 9186-7.

36. Pan D, Senpan A, Caruthers SD, Williams TA, Scott MJ, Gaffney PJ, et al. Sensitive and efficient detection of thrombus with fibrin-specific manganese nanocolloids. Chem Commun (Camb). 2009;: 3234-6.

37. Park JH, Gu L, von Maltzahn G, Ruoslahti E, Bhatia SN, Sailor MJ. Biodegradable luminescent porous silicon nanoparticles for in vivo applications. Nat Mater. 2009; 8: 331-6.

38. Tu C, Ma X, Pantazis P, Kauzlarich SM, Louie AY. Paramagnetic, silicon quantum dots for magnetic resonance and two-photon imaging of macrophages. J Am Chem Soc. 2010; 132: 2016-23.

39. Taylor KM, Rieter WJ, Lin W. Manganese-based nanoscale metal-organic frameworks for magnetic resonance imaging. J Am Chem Soc. 2008; 130: 14358-9.

40. Tan M, Lu ZR. Integrin Targeted MR Imaging. Theranostics. 2011; 1: 83-101.

41. Zhang Y, Yang Y, Cai W. Multimodality Imaging of Integrin alpha(v)beta(3) Expression. Theranostics. 2011; 1: 135-48.

42. Na HB, Lee JH, An K, Park YI, Park M, Lee IS, et al. Development of a $\mathrm{T} 1$ contrast agent for magnetic resonance imaging using $\mathrm{MnO}$ nanoparticles. Angew Chem Int Ed Engl. 2007; 46: 5397-401.

43. McCarthy JR, Weissleder R. Multifunctional magnetic nanoparticles for targeted imaging and therapy. Adv Drug Deliv Rev. 2008; 60: 1241-51.

44. Choi J, Kim K, Kim T, Liu G, Bar-Shir A, Hyeon T, et al. Multimodal imaging of sustained drug release from 3-D poly(propylene fumarate) (PPF) scaffolds. J Control Release. 2011; 156:239-45.
45. Viglianti BL, Abraham SA, Michelich CR, Yarmolenko PS, MacFall JR, Bally MB, et al. In vivo monitoring of tissue pharmacokinetics of liposome/drug using MRI: illustration of targeted delivery. Magn Reson Med. 2004; 51: 1153-62.

46. Ponce AM, Viglianti BL, Yu D, Yarmolenko PS, Michelich CR, Woo $\mathrm{J}$, et al. Magnetic resonance imaging of temperature-sensitive liposome release: drug dose painting and antitumor effects. J Natl Cancer Inst. 2007; 99: 53-63.

47. Bae KH, Lee K, Kim C, Park TG. Surface functionalized hollow manganese oxide nanoparticles for cancer targeted siRNA delivery and magnetic resonance imaging. Biomaterials. 2011; 32: 176-84.

48. Li Y, Huang TT, Carlson EJ, Melov S, Ursell PC, Olson JL, et al. Dilated cardiomyopathy and neonatal lethality in mutant mice lacking manganese superoxide dismutase. Nat Genet. 1995; 11: 376-81.

49. Gunter TE, Gavin CE, Aschner M, Gunter KK. Speciation of manganese in cells and mitochondria: a search for the proximal cause of manganese neurotoxicity. Neurotoxicology. 2006; 27: 765-76.

50. Dobson AW, Erikson KM, Aschner M. Manganese neurotoxicity. Ann N Y Acad Sci. 2004; 1012: 115-28.

51. Racette BA, Antenor JA, McGee-Minnich L, Moerlein SM, Videen TO, Kotagal V, et al. [18F]FDOPA PET and clinical features in parkinsonism due to manganism. Mov Disord. 2005; 20: 492-6.

52. Crossgrove J, Zheng W. Manganese toxicity upon overexposure. NMR Biomed. 2004; 17: 544-53.

53. Silva AC, Bock NA. Manganese-enhanced MRI: an exceptional tool in translational neuroimaging. Schizophr Bull. 2008; 34: 595-604.

54. Silva AC, Lee JH, Aoki I, Koretsky AP. Manganese-enhanced magnetic resonance imaging (MEMRI): methodological and practical considerations. NMR Biomed. 2004; 17: 532-43.

55. Aoki I, Wu YJ, Silva AC, Lynch RM, Koretsky AP. In vivo detection of neuroarchitecture in the rodent brain using manganese-enhanced MRI. Neuroimage. 2004; 22: 1046-59.

56. Lee JH, Silva AC, Merkle H, Koretsky AP. Manganese-enhanced magnetic resonance imaging of mouse brain after systemic administration of $\mathrm{MnCl}$ : dose-dependent and temporal evolution of T1 contrast. Magn Reson Med. 2005; 53: 640-8.

57. Young SW, Bradley B, Muller HH, Rubin DL. Detection of hepatic malignancies using Mn-DPDP (manganese dipyridoxal diphosphate) hepatobiliary MRI contrast agent. Magn Reson Imaging. 1990; 8: 267-76.

58. Kettritz U, Schlund JF, Wilbur K, Eisenberg LB, Semelka RC. Comparison of gadolinium chelates with manganese-DPDP for liver lesion detection and characterization: preliminary results. Magn Reson Imaging. 1996; 14: 1185-90.

59. Toft KG, Hustvedt SO, Grant D, Friisk GA, Skotland T. Metabolism of mangafodipir trisodium (MnDPDP), a new contrast medium for magnetic resonance imaging, in beagle dogs. Eur J Drug Metab Pharmacokinet. 1997; 22: 65-72.

60. Gilad AA, Walczak P, McMahon MT, Na HB, Lee JH, An K, et al. MR tracking of transplanted cells with "positive contrast" using manganese oxide nanoparticles. Magn Reson Med. 2008; 60: 1-7.

61. Yu MK, Park J, Jon S. Targeting Strategies for Multifunctional Nanoparticles in Cancer Imaging and Therapy. Theranostics. 2012; 2: 3-44.

62. Li M, Kim HS, Tian L, Yu MK, Jon S, Moon WK. Comparison of Two Ultrasmall Superparamagnetic Iron Oxides on Cytotoxicity and MR Imaging of Tumors. Theranostics. 2012; epub. 